\title{
SGLT2 Inhibitors and the Risk of Hospitalization for Fournier's Gangrene: A Nested Case-Control Study
}

\author{
Tongtong Wang (D) - Shrita M. Patel · Anne Hickman • \\ Xinyue Liu · Philip LStJ Jones · Ira Gantz • Carol E. Koro
}

Received: December 4, 2019 / Published online: February 12, 2020

(C) The Author(s) 2020

\section{ABSTRACT}

Introduction: Based on post-marketing surveillance, concern has been raised that sodiumglucose cotransporter 2 inhibitors (SGLT2i) may increase the risk of necrotizing fasciitis of the perineum (Fournier's gangrene, FG). As a result of the low incidence of FG, data from clinical trials may be insufficient to robustly assess this issue because of the relatively limited numbers of participants. Real-world evidence may help clarify the association between SGLT2i and FG in the type 2 diabetes (T2D) population.

Methods: A nested case-control study was performed using Truven Health MarketScan ${ }^{\mathrm{TM}}$ databases. Each patient with T2D hospitalized for FG between 1 April 2013 (when the first

Enhanced Digital Features To view enhanced digital features for this article go to https://doi.org/10.6084/ m9.figshare.11618133.

T. Wang $(\bowtie) \cdot X$. Liu $\cdot$ C. E. Koro

Department of Pharmacoepidemiology, Merck \& Co., Inc., Kenilworth, NJ, USA

e-mail: tongtong.wang@merck.com

S. M. Patel

Department of Clinical Safety and Risk

Management, Merck \& Co., Inc., Kenilworth, NJ, USA

A. Hickman

Safety Surveillance and Risk Management, Pfizer Inc., Groton, CT, USA
SGLT2i was available) and 31 March 2018 (latest available data) was matched (on the basis of sex, age, and cohort entry date) with six controls from the same cohort. The adjusted odds ratio (OR) of hospitalization for FG was estimated for patients receiving SGLT2i compared with those receiving two or more non-SGLT2i antihyperglycemic agents (AHAs) or insulin alone using conditional logistic regression.

Results: The cohort included 1,897,935 patients, with 216 hospitalized for FG (incidence rate, 5.2 events per 100,000 personyears). Patients with FG ranged from 23 to 79 years of age; 201 (93.1\%) were men. Among the 216 FG cases, 9 (4.2\%) were current SGLT2i users; among the 1296 matched controls, 100 $(7.7 \%)$ were current SGLT2i users. Approximately $93 \%$ of SGLT2i were used in combination. The adjusted OR of FG in patients treated with SGLT2i compared with patients treated with two or more non-SGLT2i AHAs or insulin alone was 0.55 [95\% CI 0.25-1.18].

P. LStJ Jones

Safety Surveillance and Risk Management, Pfizer Inc., Sandwich, UK

\section{Gantz}

Department of Global Clinical Development, Merck \& Co., Inc., Kenilworth, NJ, USA

C. E. Koro

Pharmaceutical Health Services Research, School of Pharmacy, University of Maryland, Baltimore, MD, USA 
Conclusion: The study did not find that treatment with SGLT2i, as compared with treatment with two or more non-SGLT2i AHAs or insulin alone, was statistically significantly associated with an increased risk of hospitalization for FG. Additional studies are needed to corroborate the findings.

Keywords: Antihyperglycemic agents; Fournier's gangrene; Nested case-control study; Sodium-glucose cotransporter 2 inhibitors (SGLT2i); Type 2 diabetes

\section{Key Summary Points}

Why carry out this study?

Based on post-marketing surveillance, concern has been raised by regulatory authorities that sodium-glucose cotransporter 2 inhibitors (SGLT2i) may increase the risk of necrotizing fasciitis of the perineum (Fournier's gangrene; FG), a rare but serious and life-threatening necrotizing infection requiring urgent surgical intervention.

Completed cardiovascular outcome trials (CVOTs) and renal outcome studies for members of the SGLT2i class may be insufficient to robustly assess this issue because of the low incidence of FG and relatively limited numbers of participants.

The nested case-control study was designed to assess whether the use of SGLT2i was associated with an increased risk of hospitalization for FG, as compared with non-SGLT2i antihyperglycemic agents (AHA) in a real-world setting.

\section{What was learned from the study?}

The study did not find that treatment with SGLT2i, as compared with treatment with two or more non-SGLT2i AHAs or insulin alone, was statistically significantly associated with an increased risk of hospitalization for FG (adjusted OR 0.55 [95\% CI 0.25-1.18]).
Although additional studies are needed to corroborate the findings, the results of the rigorous epidemiologic study are expected to provide additional context to clinicians who prescribe SGLT2 inhibitors.

\section{INTRODUCTION}

In 2018, on the basis of post-marketing adverse effects surveillance data, the European Medicines Agency (EMA) and the US Food and Drug Administration (FDA) mandated updates to the prescribing information of all members of the sodium-glucose cotransporter 2 inhibitor (SGLT2i) drug class, indicating that cases of Fournier's gangrene (FG) have been reported with the use of this class of antihyperglycemic agent (AHA) in patients with diabetes [1, 2].

FG is a rapidly progressing, necrotizing infection of the soft tissue and fascia of the perineum and genital region that requires emergency surgical intervention to debride necrotic tissue $[3,4]$. The infection is usually a synergistic polymicrobial infection, involving both aerobic and anaerobic bacteria, and treatment with broad-spectrum antibiotics is a key component of therapy $[5,6]$. Later care involves surgical reconstruction of the defects introduced by debridement [7].

Diabetes is the most common predisposing factor for FG; however, underlying disorders such as chronic alcoholism, renal failure, liver cirrhosis, and obesity have also been identified as risk factors [8-10]. Possible etiologies include cutaneous, anorectal, urogenital, and retroperitoneal causes and iatrogenic or self-inflicted trauma, among others [10, 11]. Male sex, the extremes of age (very young and old), and immunocompromised individuals are at increased risk of FG compared with other populations $[8,12]$. Reported mortality rates range from $3 \%$ to $45 \%$, with factors such as underlying comorbidities, the source of infection, and the presence of severe illness or sepsis at the time of initial evaluation affecting this outcome [8]. 
Published literature describing the occurrence of FG in men and women is limited. It has been reported that FG occurs in 1.6 out of 100,000 men annually in the USA, and most frequently occurs in men aged $50-79$ years (3.3 out of 100,000) [3]. The incidence of FG in women is extremely low, with 39 cases of FG identified from the 15.1 million women in the US State Inpatient Databases (SID, the largest US hospital care dataset) [3]. In the cardiovascular outcome trial (CVOT) for the SGLT2i dapagliflozin (DECLARE-TIMI 58), which included 17,160 participants with type 2 diabetes (T2D), six cases of FG were reported, one in the dapagliflozin group and five in the placebo group [13]. In the CVOTs for the SGLT2i empagliflozin (EMPA-REG) [14] and canagliflozin (CANVAS program) [15] and the canagliflozin renal outcome study (CREDENCE) [16], which together included a total 21,563 participants, no cases of FG were reported. However, as a result of the low incidence of FG, data from these and other ongoing SGLT2i trials may be insufficient to robustly assess this issue because of the relatively limited numbers of participants.

In the 6 years from March 2013 to January 2019, the FDA Adverse Event Reporting System (FAERS) and the medical literature identified a total of 55 cases of FG in patients taking an SGLT2i, compared with 19 cases of FG in 35 years in patients taking other (non-SGLT2i) AHAs $[2,17]$. However, FAERS data are limited, confounded by other risk factors, and subject to limitations based on spontaneous reporting.

Two recently published retrospective cohort studies compared the risk of hospitalization of FG in patients with T2D treated with SGLT2i to similar patients treated with other second-line AHAs using US claims databases [18, 19]. Dave et al. assessed the association of the risk of hospitalization for FG with initiation of SGLT2i treatment among men with T2D using three US databases. Among 138,158 SGLT2i users, there were $13 \mathrm{FG}$ cases compared with 24 cases among 360,685 dipeptidyl peptidase 4 inhibitor (DPP4i) users, corresponding to an adjusted hazard ratio of 1.73 [95\% confidence interval (CI), 0.87-3.42] [18]. Petruski-Ivleva et al. used administrative claims data from a single US database and found that the unadjusted rate ratio for FG in patients initiating SGLT2i compared to other antidiabetic medication was 0.62 (95\% CI 0.08-4.47) [19].

Further studies are warranted to clarify the association between SGLT2i and FG in the T2D population. The study reported here was designed to assess whether the use of SGLT2i was associated with an increased risk of hospitalization for FG compared with other AHAs in a real-world setting.

\section{METHODS}

\section{Study Design and Participants}

A nested case-control study was conducted within a population-based cohort identified from two US administrative claims databases: (1) Truven Health MarketScan ${ }^{\mathrm{TM}}$ Commercial Claims and Encounters Database (CCAE), a medical and drug insurance claims database of active employees, early retirees, and their dependents insured by employer-sponsored plans; and (2) Truven Health MarketScan ${ }^{\mathrm{TM}}$ Medicare Supplemental and Coordination of Benefits Database (MDCR), an administrative health claims database for Medicare-eligible active and retired employees and their Medicare-eligible dependents from employer-sponsored supplemental plans.

\section{Study Population}

All patients in the databases who were prescribed at least one AHA between April 1, 2013 (when the first SGLT2i-canagliflozin-was available in the USA) and March 31, 2018 (latest available data) and had at least one claim of T2D diagnosis during the study period were identified. Cohort entry date was defined as the date of the first prescription claim for an AHA during the study period. The AHAs considered at cohort entry were metformin, sulfonylureas (SU), thiazolidinediones (TZD), acarbose, meglitinides, DPP4i, glucagon-like peptide 1 (GLP-1) receptor agonists, SGLT2i, and insulin. The cohort was restricted to patients at least 18 years of age at the time of cohort entry who 
were continuously enrolled in the health plan for at least 6 months before cohort entry. Patients with a diagnosis of human immunodeficiency virus (HIV) infection or FG before study cohort entry were excluded. Patients with a principal diagnosis of type 1 diabetes, women with a diagnosis of gestational diabetes, and those with a principal diagnosis of polycystic ovary syndrome (for whom metformin can be prescribed) were also excluded.

Patients enrolled in the study cohort were followed from the date of cohort entry until an FG event (defined below) occurred, death, unenrollment from the health plan, or the end of the study period, whichever occurred first.

\section{Case-Control Selection}

Because of the complex time-dependent nature of AHA exposure, the large size of the cohort, and the low incidence of FG, a case-control design nested within the cohort was used. A case of FG was defined as the first hospitalization for FG, defined by the codes described below.

FG cases that occurred after October 1, 2015 were defined using the International Classification of Diseases, 10th Revision, Clinical Modification (ICD-10-CM) codes, as these codes were fully implemented in the databases by this time. FG in men was identified by inpatient claims containing ICD-10-CM diagnosis code N49.3 (Fournier gangrene) as a primary diagnosis. There is no FG diagnosis code for women. The ICD-10-CM code for FG in women is listed under N76.89 (Other specified inflammation of vagina and vulva). Because early debridement is essential $[20,21]$, to help identify female cases more accurately, we searched for patients with a primary ICD-10-CM diagnosis code of N76.89 who also have a Current Procedural Terminology (CPT) code indicating genital or perineal debridement in the anatomic area(s) of interest (Table 1). As ICD-10-CM has a designated diagnosis code that specifically defines FG in men, the CPT codes were not required to further confirm FG cases in male patients.

FG cases that occurred before October 1, 2015 were defined using the International
Table 1 CPT codes and ICD-9-PCS procedure codes for debridement

\section{Code Description}

CPT codes

11004 Debridement of skin, subcutaneous tissue, muscle, and fascia for necrotizing soft tissue infection; external genitalia and perineum

11006 Debridement of skin, subcutaneous tissue, muscle, and fascia for necrotizing soft tissue infection; external genitalia, perineum, and abdominal wall, with or without fascial closure

ICD-9-PCS procedure codes

8622 Excisional debridement of wound, infection, or
burn

CPT Current Procedural Terminology, ICD-9-PCS International Classification of Diseases, 9th Revision, Procedure Coding System

Classification of Diseases, 9th Revision, Clinical Modification (ICD-9-CM) code. Male cases of FG were identified by hospitalization claims containing ICD-9-CM code 608.83 (Vascular disorders of male genital organs) as a primary diagnosis. To identify female cases, we searched for patients with inpatient claims containing ICD-9-CM diagnosis codes for gangrene (785.4) and either abscess of Bartholin's gland (616.3) or vulvar abscess (616.4). Because there were no designated ICD-9-CM diagnosis codes for either male or female FG, all cases were required to have had a genital or perineal debridement defined by ICD-9 procedure codes or CPT codes listed in Table 1. A similar strategy was used in the observational study describing the incidence rate of FG in the US State Inpatient Databases (SID) [3].

For each hospitalization for FG occurring during the study, the date of the FG diagnosis was used to define the index date. Controls were selected from the case's risk set, which contained the cohort members being followed who did not have a diagnosis of FG at the index date. As increasing the number of controls improves the power of the study, six controls were randomly selected for each FG case patient and 
matched on the basis of sex, age ( \pm 5 years), and date of study cohort entry ( \pm 90 days) [22]. Control patients were assigned the same index date as the case patient to whom they were matched. Each case patient and the six matched controls constituted a risk stratum.

\section{Exposure Assessment}

Current AHA exposure for each patient in this study was determined by existence of AHA prescription claims whose days of supply plus a 30-day grace period included the index date. Days of supply was considered as evidence of the period in which a patient was covered for the dispensed medication in pharmacy claims [23]. Since most oral AHA prescriptions are supplied for 90 days, a 30-day grace period was selected to account for non-adherence and a potential delay in effect. In the event of late refills, dispensing with a gap shorter than the 30-day grace period was considered persistent exposure to a drug. The 30-day grace period was also added to the end of last refill to account for potential medication overstock or residual biologic effect. For both cases and controls, current exposure was hierarchically classified into the following three mutually exclusive categories: SGLT2i with or without any other AHAs (including insulin); two or more non-SGLT2i AHAs or insulin alone; and single AHAs excluding insulin or no current exposure. Since SGLT2i are considered second/third-line treatments for T2D according to the clinical guidance [24, 25], the odds ratio (OR) of hospitalization for FG in current users of SGLT2i was estimated by comparison with a reference group of patients using "two or more non-SGLT2i AHAs or insulin alone".

\section{Statistical Analysis}

Descriptive statistics were used to summarize the characteristics of the cases and matched controls. Unadjusted incidence rates of FG were calculated, and a nested case-control analysis was performed to assess the association between the use of SGLT2i and the incidence of FG hospitalization. A nested case-control analysis was used because it is computationally more efficient than a full cohort approach using a survival analysis, while producing ORs that are unbiased estimators of incidence rate ratios with little or no loss in precision [26, 27].

Conditional logistic regression was used to estimate ORs and corresponding 95\% CIs for the association between SGLT2i use and the risk of hospitalization for FG compared with use of other AHAs. In addition to conditioning the models on sex, age, and date of study cohort entry, upon which all FG cases and controls were matched, the models were adjusted for several potential confounders measured in the 6 months prior to the index date, including preexisting comorbidities and diabetic complications, insulin use (a proxy for diabetes severity), Charlson comorbidity index (CCI, a commonly used summary measure for assessing morbidity) [28], and number of hospitalizations and number of unique non-diabetic drugs (two proxies of overall health). A secondary analysis was conducted to assess the association between SGLT2i use and the risk of hospitalization for FG separately in men and women. To assess the robustness of the results, three sensitivity analyses were performed. First, to minimize the potential impact of misclassification of hospitalization for FG resulting from using less specific ICD-9-CM coding, we repeated the primary analysis including only male FG cases identified after October 1, 2015, when ICD-10$\mathrm{CM}$ codes were fully implemented in the databases. The second sensitivity analysis removed the 30-day grace period from the definition of current AHA exposure. The third sensitivity analysis compared patients receiving SGLT2i in combination with other AHAs to those treated with two or more non-SGLT2i AHAs to minimize the potential bias induced by differences among population who received SGLT2i or insulin as monotherapy.

To examine the potential effects of model over-fit for a small sample size, an additional post hoc sensitivity analysis was conducted using the reduced model that only adjusted baseline insulin use, prior hospitalization, obesity, and CCI-a summary comorbidity measure. 
All analyses were done with SAS version 9.4 (SAS Institute, Cary, NC).

\section{Compliance with Ethics Guidelines}

The database used in this study was a Health Insurance Portability and Accountability Act of 1996 (US) (HIPAA) compliant database using deidentified patient data and the study was therefore exempt from ethical approval.

\section{RESULTS}

\section{Study Population}

The cohort consisted of $1,897,935$ patients (Fig. 1), among whom 216 had been hospitalized for FG during 4,163,536 person-years of follow-up (crude incidence rate, 5.2 events per 100,000 person-years). Among the 1,005,561 male patients in the cohort, 201 had been hospitalized for FG (crude incidence rate, 9.2 events per 100,000 person-years). Among the 892,374 female patients in the cohort, 15 had been hospitalized for FG (crude incidence rate, 0.8 events per 100,000 person-years).

The baseline characteristics of the 216 FG cases and 1296 matched controls (non-FG cases) are shown in Table 2 . As a result of matching, cases and controls had the same distribution in terms of sex, age, calendar year of study entry, and duration of follow-up; approximately 93\% of patients were male, mean age was 56 years, and mean duration of follow-up was 2.1 years.

At the index date of FG diagnosis, FG cases had a higher prevalence of obesity than matched controls $(28.7 \%$ vs. $15.0 \%)$. The prevalence of insulin use was also higher among FG cases than among matched controls (38.9\% vs. $17.6 \%)$. Compared with matched controls, FG cases had a higher prevalence of cardiovascular diseases, including coronary heart disease, coronary heart failure, and peripheral artery disease. Similarly, the prevalence of diabetesrelated complications (diabetic ketoacidosis, diabetic nephropathy, diabetic neuropathy, and diabetic retinopathy) was also higher among FG cases than among matched controls.
Additionally, FG cases were more likely to be hospitalized within 6 months prior to the event (index date) than were matched controls prior to that date $(24.5 \%$ vs. $5.5 \%)$.

\section{SGLT2i and Hospitalization for FG}

Table 3 summarizes current exposure to AHAs (as defined in "Methods") among 216 FG cases and 1296 matched controls in the three mutually exclusive categories of drug exposure. At the index date, $4.2 \%$ of FG cases used SGLT2i, $44.9 \%$ used two or more non-SGLT2i AHAs or insulin alone, and 50.9\% used AHA monotherapy excluding insulin or were without current exposure; corresponding values for controls were $7.7 \%, 36.6 \%$, and $55.7 \%$, respectively. Approximately $93 \%$ of SGLT2i users were in combination with other AHAs or insulin (7/9 SGLT2i users among FG cases and 94/100 SGLT2i users among controls). The adjusted OR of FG in patients treated with SGLT2i compared to patients treated with two or more nonSGLT2i AHAs or insulin alone was 0.55 [95\% CI 0.25-1.18], after adjusting for multiple confounding factors (Table 4).

Similar results were obtained when the study cohort was stratified by sex. Among the 201 male FG cases, 8 (4.0\%) were current SGLT2i users, and among the 1206 matched controls, 85 (7.1\%) were current SGLT2i users (Table 3). The adjusted OR of hospitalization for FG in current users of SGLT2i compared to users of two or more non-SGLT2i AHAs or insulin alone was 0.63 (95\% CI 0.28-1.42) in male patients with T2D, after adjusting for multiple confounding factors (Table 4).

Fifteen women with T2D met the case definition for FG. Among those, only one used an SGLT2i at the index date. Among the 90 matched controls, 15 (16.7\%) were current SGLT2i users (Table 3). As a result of the low yield, only descriptive analysis was conducted for female FG cases.

\section{Sensitivity Analyses}

Sensitivity analyses gave results that were consistent with those of the primary analyses. 


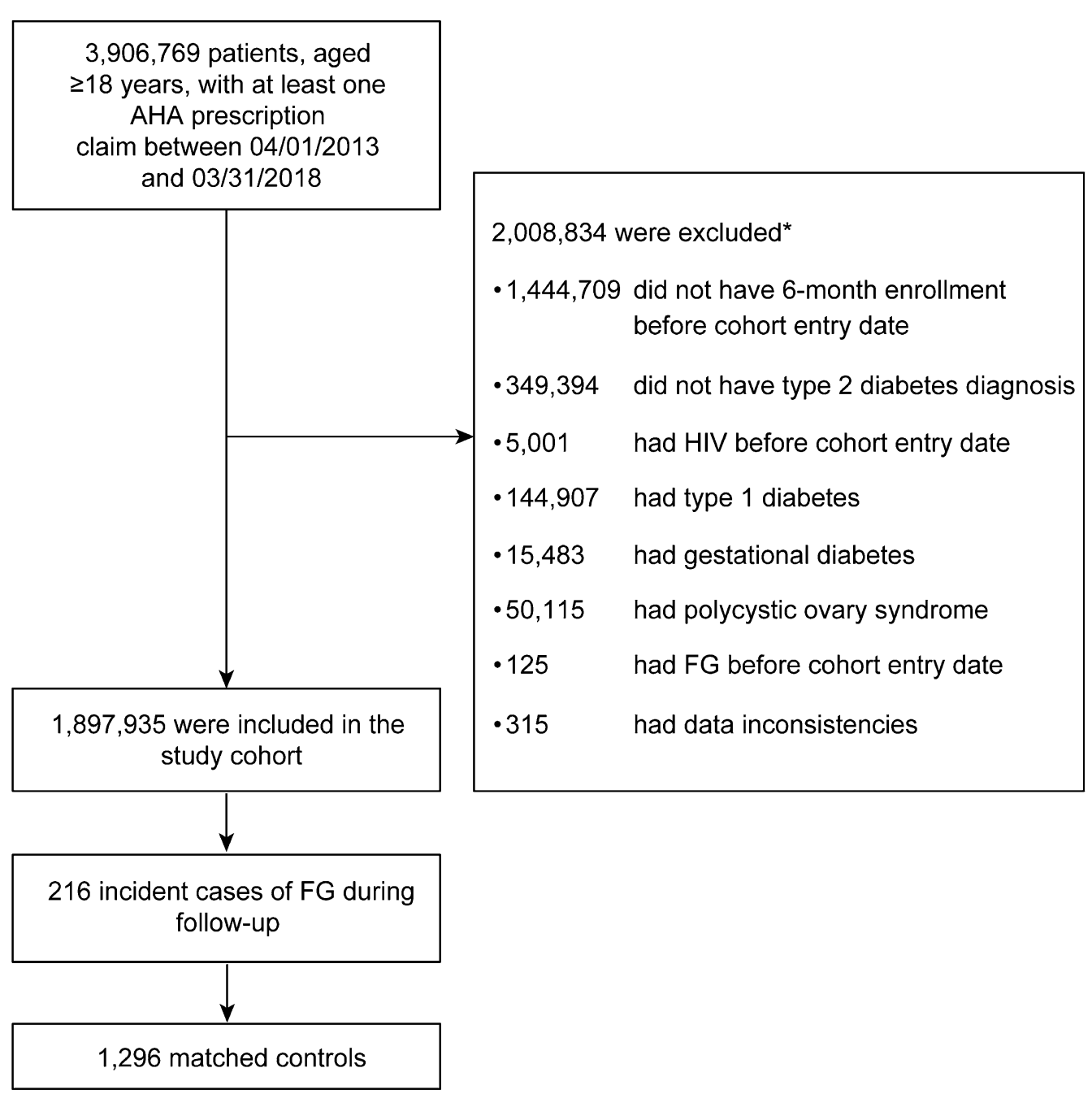

*A patient may be excluded for more than 1 reason.

Fig. 1 Flowchart of patient selection

Similar results were observed with respect to the risk of hospitalization for FG among patients with T2D when a sensitivity analysis was conducted by removing the 30-day grace period from the definition of current AHA exposure (adjusted OR in users of SGLT2i vs. users of two or more non-SGLT2i AHAs or insulin alone, 0.56; 95\% CI 0.24-1.32). The results were also similar when only male FG cases identified after October 1, 2015, when ICD-10-CM codes were fully implemented in the databases and specifically captured a diagnosis of FG, were included (adjusted OR in users of SGLT2i vs. users of two or more non-SGLT2i AHAs or insulin alone,
0.84; 95\% CI 0.35-2.02). Furthermore, the adjusted OR in users of SGLT2i in combination with other AHAs vs. users of two or more nonSGLT2i AHAs was 0.48 (95\% CI 0.20-1.14) when patients who received SGLT2i or insulin as monotherapy were excluded from the primary analysis (Table 4).

The post hoc analysis using a reduced conditional logistic regression model produced results that were also consistent with the primary analysis (adjusted OR in users of SGLT2i vs. users of two or more non-SGLT2i AHAs or insulin alone, 0.56 ; 95\% CI 0.26-1.20). 
Table 2 Baseline characteristics of patients with type 2 diabetes who were hospitalized for Fournier's gangrene (cases) and matched controls at the index date of FG diagnosis

\begin{tabular}{|c|c|c|}
\hline Characteristics & Cases & Controls \\
\hline$N$ & 216 & 1296 \\
\hline Male, $n(\%)$ & $201(93.1)$ & $1206(93.1)$ \\
\hline Age, mean $\pm S D$ & $56.2 \pm 10.9$ & $56.4 \pm 10.5$ \\
\hline $\begin{array}{l}\text { Duration of follow-up } \\
\text { (days), mean } \pm S D\end{array}$ & $774.6 \pm 506.5$ & $774.7 \pm 506.8$ \\
\hline Hypertension, $n(\%)$ & $137(63.4)$ & $771(59.5)$ \\
\hline Obesity, $n(\%)^{* *}$ & $62(28.7)$ & $195(15.0)$ \\
\hline Insulin use, $n(\%)^{* *}$ & $84(38.9)$ & $228(17.6)$ \\
\hline $\begin{array}{l}\text { Coronary heart disease, } \\
n(\%)^{*}\end{array}$ & $39(18.1)$ & $157(12.1)$ \\
\hline $\begin{array}{l}\text { Congestive heart failure, } \\
\left(n(\%)^{* *}\right.\end{array}$ & $31(14.4)$ & $42(3.2)$ \\
\hline $\begin{array}{l}\text { Peripheral artery disease, } \\
n(\%)^{* *}\end{array}$ & $27(12.5)$ & $52(4.0)$ \\
\hline Stroke, $n(\%)$ & $4(1.9)$ & $21(1.6)$ \\
\hline $\begin{array}{l}\text { Lower extremity } \\
\text { amputation, } n(\%)\end{array}$ & $2(1.0)$ & $4(0.3)$ \\
\hline $\begin{array}{l}\text { Diabetic ketoacidosis, } \\
n(\%)^{*}\end{array}$ & $4(1.9)$ & $3(0.2)$ \\
\hline $\begin{array}{l}\text { Diabetic neuropathy, } \\
n(\%)^{* *}\end{array}$ & $39(18.1)$ & $111(8.6)$ \\
\hline $\begin{array}{l}\text { Diabetic retinopathy, } \\
n(\%)^{*}\end{array}$ & $15(6.9)$ & $44(3.4)$ \\
\hline Pancreatitis, $n(\%)^{*}$ & $6(2.8)$ & $6(0.5)$ \\
\hline $\begin{array}{l}\text { Moderate to severe } \\
\text { renal } \\
\text { insufficiency/diabetic } \\
\text { nephropathy, } n(\%)^{* *}\end{array}$ & $40(18.5)$ & $92(7.1)$ \\
\hline \multicolumn{3}{|c|}{ Charlson comorbidity index, $n(\%)^{* *}$} \\
\hline 0 & $1(0.5)$ & $211(16.3)$ \\
\hline 1 & $73(33.8)$ & $675(52.1)$ \\
\hline$\geq 2$ & $142(65.7)$ & $410(31.6)$ \\
\hline
\end{tabular}

Table 2 continued

\begin{tabular}{lll}
\hline Characteristics & Cases & Controls \\
\hline $\begin{array}{l}\geq 6 \text { of non-diabetic } \\
\text { medications, } n(\%)^{*}\end{array}$ & $130(60.2)$ & $579(44.7)$ \\
\multicolumn{2}{l}{ No. of non-insulin antidiabetic medications, $n(\%)^{* *}$} \\
0 & $75(34.7)$ & $236(18.2)$ \\
1 & $76(35.2)$ & $624(48.2)$ \\
$\geq 2$ & $65(30.1)$ & $436(33.6)$ \\
Prior hospitalizations, & $53(24.5)$ & $70(5.5)$ \\
$n(\%)^{* *}$ & & \\
\hline
\end{tabular}

$S D$ standard deviation

${ }^{*} p<0.05$ for difference between cases and controls; ${ }^{* *} p<0.001$ for difference between cases and controls

\section{DISCUSSION}

This observational study examined the effect of SGLT2i on the risk of hospitalization for FG among patients with T2D seen in routine clinical practice. The study did not find that treatment with SGLT2i, as compared with treatment with two or more non-SGLT2i AHAs or insulin alone, was statistically significantly associated with an increased risk of hospitalization for FG (adjusted OR $=0.55 ; 95 \%$ CI 0.25-1.18). Similar results were obtained when male and female patients were analyzed separately, and the results were supported by all sensitivity analyses.

The findings of this study are consistent with the evidence from the prospective, randomized, placebo-controlled DECLARE-TIMI 58 trial, which found no association between dapagliflozin and the risk of hospitalization for FG [13]. The findings are also consistent with the evidence from the observational study conducted by Petruski-Ivleva et al. which found no increased risk of FG among SGLT2i users compared to similar patients treated with other second-line AHAs, although the small number of events yielded wide confidence intervals in this observational investigation [19]. 
Table 3 Current AHA use among patients with type 2 diabetes who were hospitalized for Fournier's gangrene (cases) and matched controls

\begin{tabular}{|c|c|c|c|c|c|c|}
\hline \multirow[t]{2}{*}{ Treatment $^{\mathrm{a}}$} & \multicolumn{2}{|l|}{ Overall } & \multicolumn{2}{|c|}{ Male patients } & \multicolumn{2}{|c|}{ Female patients } \\
\hline & $\begin{array}{l}\text { Cases } \\
(N=216)\end{array}$ & $\begin{array}{l}\text { Controls } \\
(N=1296)\end{array}$ & $\begin{array}{l}\text { Cases } \\
(N=201)\end{array}$ & $\begin{array}{l}\text { Controls } \\
(N=1206)\end{array}$ & $\begin{array}{l}\text { Cases } \\
(N=15)\end{array}$ & $\begin{array}{l}\text { Controls } \\
(\boldsymbol{N}=90)\end{array}$ \\
\hline $\begin{array}{l}\text { SGLT2i (with or without other } \\
\text { AHAs) }\end{array}$ & $9(4.2 \%)$ & $100(7.7 \%)$ & $8(4.0 \%)$ & $85(7.1 \%)$ & $1(6.7 \%)$ & $15(16.7 \%)$ \\
\hline $\begin{array}{l}\text { Two or more non-SGLT2i AHAs } \\
\text { or insulin alone }\end{array}$ & 97 (44.9\%) & $474(36.6 \%)$ & $89(44.3 \%)$ & $447(37.1 \%)$ & $8(53.3 \%)$ & $27(30.0 \%)$ \\
\hline $\begin{array}{l}\text { Two or more non-SGLT2i } \\
\text { AHAs }\end{array}$ & $59(27.3 \%)$ & $411(31.7 \%)$ & $55(27.4 \%)$ & 385 (31.9\%) & $4(26.7 \%)$ & $26(28.9 \%)$ \\
\hline Insulin alone & $38(17.6 \%)$ & $63(4.9 \%)$ & $34(16.9 \%)$ & $62(5.1 \%)$ & $4(26.7 \%)$ & $1(1.1 \%)$ \\
\hline $\begin{array}{l}\text { Single AHAs excluding insulin or } \\
\text { no current exposure }\end{array}$ & $110(50.9 \%)$ & $722(55.7 \%)$ & $\begin{array}{l}104 \\
\quad(51.7 \%)\end{array}$ & $674(55.9 \%)$ & $6(40.0 \%)$ & $48(53.3 \%)$ \\
\hline
\end{tabular}

${ }^{a}$ As defined in "Methods"

In contrast, a recently reported retrospective cohort study, conducted by Dave et al., compared FG risk in patients treated with SGLT2i or DPP4i among men with T2D and reported a numerically increased risk of hospitalization for FG associated with SGLT2i which was not statistically significant (adjusted hazard ratio 1.73; 95\% CI 0.87-3.42) [18]. Compared with the present study, use of less specific ICD-9 codes by Dave et al. could have resulted in a greater potential for misclassification of FG. Furthermore, the present study provides a more comprehensive assessment of the risk of FG by including data from patients with T2D treated with various AHAs, compared to the Dave et al. study, which only included incretin-based therapy as comparison.

A recent analysis of post-marketing, spontaneous case reports revealed 55 cases of FG in patients using SGLT2i over a nearly 6-year period between March 2013 and January 2019 compared with only 19 cases in patients using other diabetes medications over a 35-year period [17]. Approximately $78 \%$ of the post-marketing, spontaneously reported cases (43/55) were submitted after the initial analysis upon which the safety warning was based [2], suggesting the possibility of stimulated reporting of FG cases in patients receiving SGLT2i. Given the larger number of cases of FG identified in patients receiving non-SGLT2i AHAs in the study reported here, underreporting of postmarketing adverse events in patients receiving non-SGLT2i AHAs is also likely. Furthermore, the imbalance described in the analysis of postmarketing surveillance data could also be attributed to the Weber effect, which is a phenomenon of increased volume of reported adverse events for new drugs within their first years of approval, as health care professionals tend to report adverse events with new treatments but not with older drugs [29].

This study has several strengths. Having access to data from 1.9 million patients with T2D and 4.2 million person-years of observation, a large number of FG cases from two US claims databases were identified. The observed incidence rate of FG among male patients with T2D of 9.2 per 100,000 person-years is higher than the general population rate of 1.6 in 100,000 men per year [3], which is supportive of published literature indicating T2D as a risk factor for FG. Furthermore, the data are consistent with previously published literature with respect to the important known risk factors for FG, including obesity, diabetes severity, poor overall health, heart disease, and renal insufficiency $[8,9]$. 


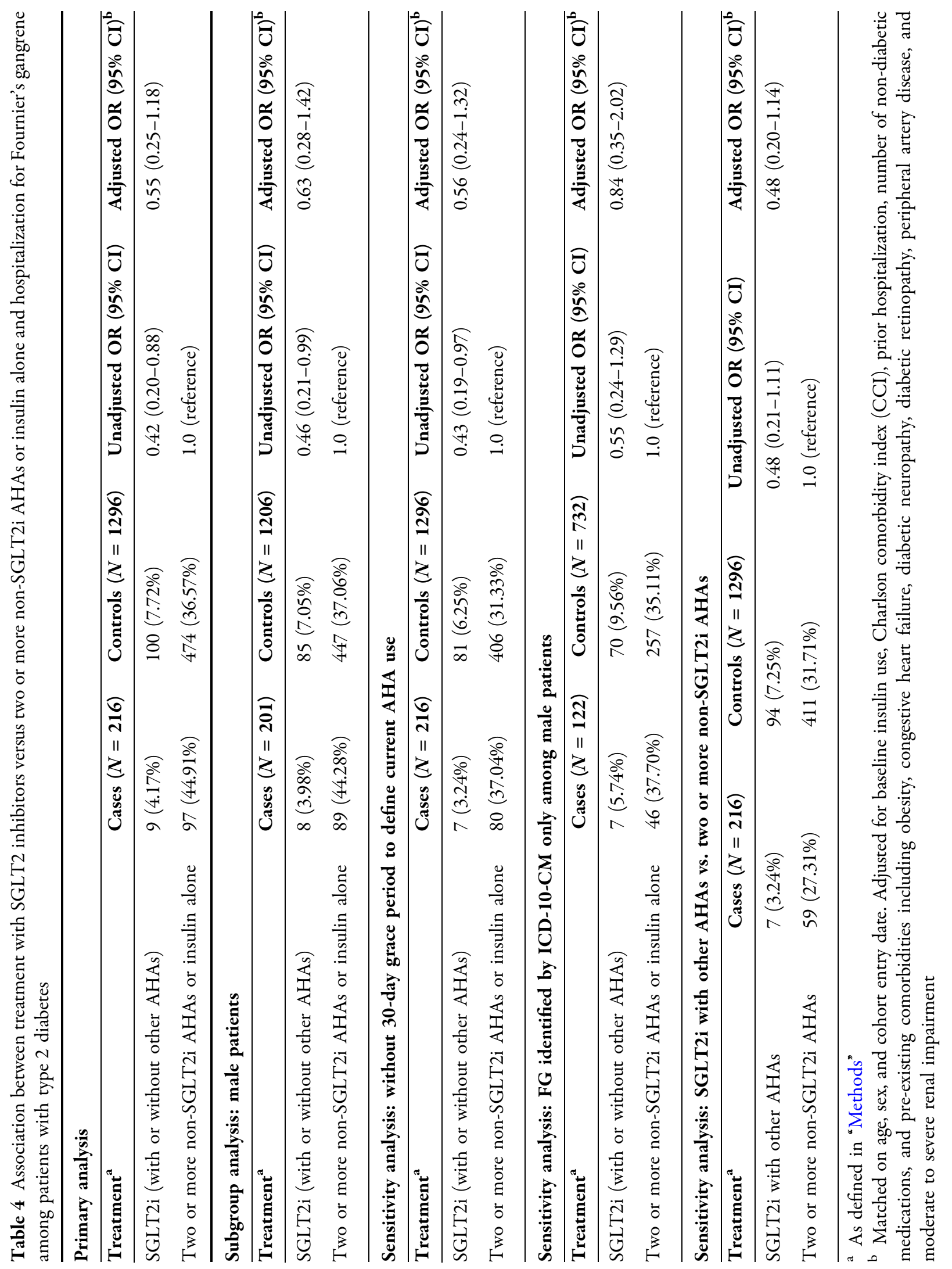


Nesting the FG cases and controls within a cohort of T2D controlled the effect of T2D on the outcome. Although the study is observational in nature and thus susceptible to potential confounding, rigorous matching and statistical adjustment were used to minimize confounding. The choice of a primary reference group of patients receiving treatment with combinations of two or more non-SGLT2i AHAs or insulin alone provided a clinically relevant treatment comparison and helped reduce potential confounding by indication.

The study is observational in nature and thus susceptible to several limitations. The first relates to the validity of the diagnosis of FG. While there is a designated ICD-10-CM diagnosis code to help identify male FG cases, the ICD-10-CM code for female FG cases and ICD-9$\mathrm{CM}$ code for both male and female FG cases are less specific. This may lead to misclassification of FG cases. However, to reduce bias due to misclassification in the absence of a designated diagnosis code, the search used for this analysis included terms for patients using both a diagnosis code and a genital or perineal debridement procedure code, as surgical management is the cornerstone of treatment for FG. A similar strategy was previously used to help identify FG cases in the US State Inpatient Databases [3]. Further, a sensitivity analysis including only male FG cases identified after October 1, 2015, when ICD-10-CM codes were fully implemented and specifically captured a diagnosis of FG, produced results that were consistent with those of the primary analysis.

Drug exposure in both cases and matched controls was based on prescriptions that were filled at outpatient pharmacies, not those prescribed or administered within inpatient services, although it is unlikely that inpatient dispensing would have accounted for a large proportion of prescriptions. It is also not known whether medications were taken as directed, although repeated dispensing of the same drug would suggest that this was the case.

Furthermore, this analysis is unable to directly adjust for the level of glycemic control in cases and controls because $\mathrm{HbA}_{1 \mathrm{C}}$ measurements are sparsely captured in the MarketScan ${ }^{\mathrm{TM}}$ databases. Because of this drawback, it is difficult to determine whether the duration of diabetes, the lack of glycemic control, or both may be associated with FG. Additionally, potential residual confounding by behavioral factors that are not well captured in claims databases, such as smoking or alcohol consumption, cannot be ruled out. However, the risk estimates were adjusted for all available potential confounding factors, such as the multisource comorbidity index, various diabetes-related complications, and the use of insulin (a proxy for advanced disease). Additionally, the choice of a primary reference group of patients receiving treatment with combinations of two or more non-SGLT2i AHAs or insulin alone further reduces potential confounding by disease severity.

Although Truven Health MarketScan ${ }^{\mathrm{TM}}$ databases are expected to be largely representative of the commercially insured population in the USA, they may not be representative of the entire US treated population, such as the uninsured or elderly people with Medicare insurance. As such, the study results are generalizable only to the commercial health insurance population from which the study population was derived and others with similar characteristics.

Finally, despite a total of 216 FG events identified from the study cohort, only nine used SGLT2i. The small number of exposed events yielded wide confidence intervals that preclude a definitive conclusion.

\section{CONCLUSIONS}

In the analysis of a large cohort of patients with T2D from two US claims databases, treatment with SGLT2i, as compared with treatment with two or more non-SGLT2i AHAs or insulin alone, was not statistically significantly associated with an increased risk of hospitalization for FG. Additional studies are needed to corroborate these findings.

\section{ACKNOWLEDGEMENTS}

Funding. This analysis and the Rapid Service Fee were funded by Merck Sharp \& Dohme 
Corp., a subsidiary of Merck \& Co., Inc., Kenilworth, NJ, USA and Pfizer Inc., New York, NY, USA.

Editorial Assistance. Editorial assistance was provided by Jennifer Rotonda, $\mathrm{PhD}$, and Edward A. O'Neill, PhD, of Merck \& Co., Inc., Kenilworth, NJ, USA. This assistance was funded by Merck \& Co., Inc., Kenilworth, NJ, USA.

Authorship. All named authors meet the International Committee of Medical Journal Editors (ICMJE) criteria for authorship for this article, take responsibility for the integrity of the work as a whole, and have given their approval for this version to be published.

Disclosures. Tongtong Wang, Shrita M. Patel, Xinyue Liu, Ira Gantz and Carol E. Koro are employees of Merck Sharp \& Dohme Corp., a subsidiary of Merck \& Co., Inc., Kenilworth, NJ, USA, and may own stock and/or hold stock options in the company. Anne Hickman and Philip Jones are employees of Pfizer Inc., New York, NY, USA and may own stock and/or hold stock options in the company.

Compliance with Ethics Guidelines. The database used in this study was a Health Insurance Portability and Accountability Act of 1996 (US) (HIPAA) compliant database using deidentified patient data and the study was therefore exempt from ethical approval.

Data Availability. Data used in this analysis were extracted from the Truven Health MarketScan ${ }^{\mathrm{TM}}$ Commercial Claims and Encounters Database and Truven Health MarketScan ${ }^{\mathrm{TM}}$ Medicare Supplemental and Coordination of Benefits Database (owned by Truven Health Analytics, Ann Arbor, MI, USA) on April 15, 2019.

Open Access. This article is distributed under the terms of the Creative Commons Attribution-NonCommercial 4.0 International License (http://creativecommons.org/licenses/ by-nc/4.0/), which permits any noncommercial use, distribution, and reproduction in any medium, provided you give appropriate credit to the original author(s) and the source, provide a link to the Creative Commons license, and indicate if changes were made.

\section{REFERENCES}

1. European Medicines Agency. PRAC recommendations on signals. Adopted at the 26-29 November 2018 PRAC meeting. https://www.ema.europa.eu/ en/documents/other/new-product-informationwording-extracts-prac-recommendations-signalsadopted-26-29-november-2018_en.pdf. Accessed July 14, 2019.

2. US Food and Drug Administration. FDA warns about rare occurrences of a serious infection of the genital area with SGLT2 inhibitors for diabetes. https://www.fda.gov/Drugs/DrugSafety/ ucm617360.htm. Accessed July 14, 2019.

3. Sorensen MD, Krieger JN. Fournier's gangrene: epidemiology and outcomes in the general US population. Urol Int. 2016;97(3):249-59.

4. Shyam DC, Rapsang AG. Fournier's gangrene. Surgeon. 2013;11(4):222-32.

5. Yilmazlar T, Gulcu B, Isik O, Ozturk E. Microbiological aspects of Fournier's gangrene. Int J Surg. 2017;40:135-8.

6. Stevens DL, Bisno AL, Chambers HF, et al. Practice guidelines for the diagnosis and management of skin and soft tissue infections: 2014 update by the Infectious Diseases Society of America. Clin Infect Dis. 2014;59(2):147-59.

7. Karian LS, Chung SY, Lee ES. Reconstruction of defects after Fournier gangrene: a systematic review. Eplasty. 2015;15:e18.

8. Eke N. Fournier's gangrene: a review of 1726 cases. Br J Surg. 2000;87(6):718-28.

9. Chinchilla RM, Morejon EI, Pietricica BN, Franco EP, Albasini JLA, Lopez BM. Fournier's gangrene. Descriptive analysis of 20 cases and literature review. Actas Urol Esp. 2009;33(8):873-80.

10. Heyns CF. Theron PD. Fournier's gangrene. In: Hohenfellner M, Santucci RA, editors. Emergencies in urology. Berlin: Springer; 2007.

11. Pais VM, Santora TA, Rukstalis DB, Sollender GE. https://emedicine.medscape.com/article/2028899overview. Accessed July 14, 2019. 
12. Rodríguez Alonso A, Pérez García MD, Núñez López A, et al. Fournier's gangrene: anatomo-clinical features in adults and children. Therapy update. Actas Urol Esp. 2000;24(4):294-306.

13. Wiviott SD, Raz I, Bonaca MP, et al. Dapagliflozin and cardiovascular outcomes in type 2 diabetes. N Engl J Med. 2019;380(4):347-57.

14. Zinman B, Wanner C, Lachin JM, et al. Empagliflozin, cardiovascular outcomes, and mortality in type 2 diabetes. N Engl J Med. 2015;373(22): 2117-28.

15. Neal B, Perkovic V, Mahaffey KW, et al. Canagliflozin and cardiovascular and renal events in type 2 diabetes. N Engl J Med. 2017;377(7):644-57.

16. Perkovic V, Jardine MJ, Neal B, et al. Canagliflozin and renal outcomes in type 2 diabetes and nephropathy. N Engl J Med. 2019;380(24): 2295-306.

17. Bersoff-Matcha SJ, Chamberlain C, Cao C, Kortepeter $\mathrm{C}$, Chong WH. Fournier gangrene associated with sodium-glucose cotransporter- 2 inhibitors: a review of spontaneous postmarketing cases. Ann Intern Med. 2019;170(11):764-9.

18. Dave CV, Schneeweiss S, Patorno E. Association of sodium-glucose cotransporter 2 inhibitor treatment with risk of hospitalization for Fournier gangrene among men. JAMA Intern Med. 2019;179(11): 1587-90.

19. Petruski-Ivleva N, Schneeweiss S, Eapen S, Rajan A, Jan S. Fournier's gangrene in patients with type 2 diabetes using second-line antidiabetic medications. Diabetes Obes Metab. 2019. https://doi.org/ 10.1111/dom.13886.

20. Chia L, Crum-Cianflone NF. Emergence of multidrug resistant organisms (MDROs) causing Fournier's gangrene. J Infect. 2018;76(1):38-43.
21. Tang LM, Su YJ, Lai YC. The evaluation of microbiology and prognosis of Fournier's gangrene in past five years. SpringerPlus. 2015;4:14.

22. Grimes DA, Schulz KF. Compared to what? Finding controls for case-control studies. Lancet. 2005;365(9468):1429-33.

23. Nielsen LH, Lokkegaard E, Andreasen AH, Keiding N. Using prescription registries to define continuous drug use: how to fill gaps between prescriptions. Pharmacoepidemiol Drug Saf. 2008;17(4):384-8.

24. Davies MJ, D’Alessio DA, Fradkin J, et al. Management of hyperglycemia in type 2 diabetes, 2018. A consensus report by the American Diabetes Association (ADA) and the European Association for the Study of Diabetes (EASD). Diabetes Care. 2018;41(12):2669-701.

25. American Diabetes Association. 9. Pharmacologic approaches to glycemic treatment: standards of medical care in diabetes-2019. Diabetes Care. 2019;42(Suppl 1):S90-102.

26. Suissa S. Novel approaches to pharmacoepidemiology study design and statistical analysis. In: Strom BL, editor. Pharmacoepidemiology. 4th ed. New York: Wiley; 2005. p. 811-29.

27. Essebag V, Platt RW, Abrahamowicz M, Pilote L. Comparison of nested case-control and survival analysis methodologies for analysis of time-dependent exposure. BMC Med Res Methodol. 2005;5(1): 5 .

28. Quan H, Li B, Couris CM, et al. Updating and validating the Charlson comorbidity index and score for risk adjustment in hospital discharge abstracts using data from 6 countries. Am J Epidemiol. 2011;173(6):676-82.

29. Weiss-Smith S, Deshpande G, Chung S, Gogolak V. The FDA drug safety surveillance program: adverse event reporting trends. Arch Intern Med. 2011;171(6):591-3. 